\title{
ras Mutations and a cup of coffee: cause, confounder, effect modifier, or what else?
}

We should not be surprised by the complexity of the relations we tackle in biology, medicine and public health. A reason for such complexity is the phenomenon of parastasis - that is, the fact that the living organism can accomplish the same end by different pathways; this is an elementary, evolutionary mechanism by which organisms have defended themselves against the environmental threats. However, according to a strongly rooted tradition, we tend to believe only or preferentially in simple cause-effect relations, those that we find in the simplest interactions we have with everyday's life objects. Medicine, in particular, tends to oversimplify causes because it aims to prevent or to cure - that is, to eliminate the causal agent.

In the specific case of the interesting paper by Miquel Porta and colleagues, ${ }^{1}$ we would be tempted to say that coffee consumption causes pancreatic cancer via ras mutations. This would be the "happy and simple end" of a long and frustrating story. Apart from tobacco smoking, we know virtually nothing about the causes of this highly aggressive cancer, which is almost incurable. So, when Professor MacMahon from Harvard in 1981 published a paper in the New England Fournal of Medicine, claiming that high consumption of coffee increased the risk of pancreatic cancer, many people thought that a new, genuine risk factor had been discovered. ${ }^{2}$ Biological plausibility was satisfied, as some researchers showed that coffee was able to induce mutations in some strains of bacteria. If we had frozen the story at that time, and thawed it now, we would probably have a sense of accomplishment, as the paper by Porta and colleagues seems to give us a further piece of evidence: that is, heavy coffee drinkers with pancreatic cancer show a greater proportion of mutations of the ras cancer gene than patients who do not drink coffee.

Well, this is not the way the story should be told, and it is not told like that by Porta and colleagues, who must be congratulated for their clarity, prudence, and intellectual honesty. Firstly, epidemiological studies do not support a link between coffee consumption (the most common beverage in the world) and pancreatic cancer. The studies that showed an association were probably confounded by tobacco smoking, as heavy coffee consumers tend also to be heavy smokers, and were likely to be affected by other types of bias. Secondly, in the paper in question there is no mutational spectrum that might suggest a causal pathway linking coffee, ras mutations and this type of cancer. Thirdly, alternative explanations are more likely, and they might prove to be even more fascinating.

Before describing the alternative explanations that Porta and colleagues put forward, I want to jump into a totally different field, social sciences. According to Robert Jervis, the author of System effects, ${ }^{3}$ complex systems such as human societies have three main characteristics. Firstly, in addition to direct effects we observe indirect and delayed effects. Secondly, when there are more than two actors in a system, the relations between any two (say, coffee and cancer) will depend by the interactions among the other members. Thirdly, relations in a system are interactive rather than additive (that is, we have positive or negative feedbacks). In international politics, the classic example of negative feedback is the balance of power: small states tend to join forces so as not to be dominated by stronger ones. Assimilating natural and social sciences is always a hazard, and may be extremely misleading. However, I believe that Jervis' three points bear more than superficial similarities to many biological situations.

In fact, what Porta and colleagues may have just opened is the realm of effect modifications - that is, of exposures that act neither as direct causes nor as confounders, but just by making the action of some genuine cause easier and more powerful. Smoking is likely to be a direct cause of pancreatic cancer, and the confounder for several exposures that have been investigated. Coffee is likely to be different. According to Porta and colleagues, coffee might work in two different ways. One is the induction or inhibition of metabolic pathways that, in turn, are involved in the activation or inactivation of carcinogenic chemicals. Or, coffee can inhibit the relevant DNA repair mechanisms, a possibility that is suggested by a wealth of molecular, cellular and animal studies. There is evidence in support of each interpretation, and the two are not mutually exclusive. Personally, I favour the second one, both because the evidence seems to be be rather convincing, and because it would explain the lack of a mutational spectrum that could be in theory attributed to the action of a single, specific carcinogen. So, coffee abstainers might be protected from ras mutated cancer thanks to a better capacity to repair DNA damage.

We can further expand the reasoning by Porta and colleagues by saying that cancer is a consequence of cumulative unrepaired DNA damage, a concept that Marianne Berwick and I have entertained in a review on the "mutagen sensitivity assays"(unpublished data). Instead of limiting ourselves to identify single, powerful and direct carcinogens, we may admit that cancer also arises as the consequence of many low dose exposures that overcome the DNA repair capacity of the individual. Like in microbiology with the concept of balance between the immunological response and the viral/bacterial assault, similarly in carcinogenesis we may admit a balance between variable repair capacity and external genotoxic exposures. I would not be surprised if "molecular epidemiology" becomes the field of research in which effect modification is the rule rather than the exception. Clearly, this makes things more complicated, as it implies the need for more statistical power to detect interactions, rather than simple additive effects. ${ }^{4}$ Longitudinal designs with repeated measures are also needed to disentangle complex temporal relations. The paper by Porta and colleagues is a good starting point for discussion.

University of Torino, Italy

PAOLO VINEIS

1 Porta M, Malats N, Guarner L, et al, for the PANKRAS II Study Group. Association between coffee drinking and K-ras mutations in exocrine pancreatic cancer. F Epidemiol Community Health 1999;53:702-9.

2 MacMahon B, Yen S, Trichopoulos D, et al. Coffee and cancer of the pancreas. N Engl f Med 1981;304:630-3.

3 Jervis R. System effects. Princeton: Princeton University Press, 1998.

4 Gracia-Closas M, Lubin JH. Power and sample size calculations in case-control studies of gene-environment interactions: comments on different approaches. Am f Epidemiol 1999;149:689-92. 